\title{
Determinación de límites de transmisión en sistemas eléctricos de potencia
}

\section{Determination of Transmission Limits on Electric Power Systems}

\author{
Castellanos-Bustamante Rafael \\ Instituto de Investigaciones Eléctricas \\ Correo:rcb@iie.org.mx
}

Información del artículo: recibido: enero de 2013, aceptado: abril de 2013

\begin{abstract}
Resumen
En este artículo se presenta la aplicación de varias metodologías para calcular el límite de transferencia de potencia que garantice una operación segura en las redes de energía eléctrica. Se describen aspectos de interés para la determinación de flujos máximos de energía eléctrica a través de líneas de transmisión en redes eléctricas. Se determina el límite térmico de conductores eléctricos, la cargabilidad de líneas de transmisión y el límite de estabilidad ante oscilaciones interárea de enlaces de transmisión entre regiones. Para el caso de oscilaciones interárea, se presentan los resultados de la aplicación de un esquema de corte de carga para estabilizar un modo de oscilación interárea al amortiguar oscilaciones negativas y llevar con ello al sistema eléctrico a una condición de operación estable. Para ilustrar la metodología se utiliza un modelo del sistema interconectado mexicano.
\end{abstract}

\footnotetext{
Abstract

This article provides the application of several methodologies to obtain power transmission limits through interties of the electric power systems to obtain a secure operation of the energy power system. Several aspects to obtain the maximum power flows of electrical energy thought of transmission lines on electrical grids are shown. Thermal limit of electrical conductors, loadability limit of transmission lines and small signal stability limits are obtained for several interties between geographical regions. It also, shows the application of a load shedding scheme to stabilize an interarea oscillation mode. The model of the Mexican Interconnected power System is used to illustrate these methodologies.
}

\section{Descriptores:}

- límites de transmisión

- límite térmico

- cargabilidad de líneas

- límite oscilaciones ante disturbios pequeños

\section{Keywords:}

- transmission limits

- thermal limit

- loadability of lines

- small signal oscillation limit 


\section{Introducción}

La determinación de límites de transferencia de energía eléctrica es una tarea fundamental para garantizar la seguridad de los sistemas de energía eléctrica. Existen diversas restricciones que limitan el flujo de energía eléctrica a través de líneas de transmisión. Desde el punto de vista físico, la máxima transferencia de energía por un conductor se obtiene mediante la determinación de la relación corriente-temperatura, llamada limite térmico (IEEE Std. 738-2002). Sin embargo, existen otras restricciones que en muchos casos no permiten la operación de las líneas eléctricas con flujos de potencia cercanos a su límite térmico, tal es el caso de límites de cargabilidad, disturbios severos, bajo voltaje, disturbios pequeños y baja frecuencia (Kundur, 1994; Westinghouse Ekectric Corporation, 1950).

En este artículo se aplican metodologías para calcular el límite de transferencia de potencia que garantice una operación segura tanto en condiciones de prefalla como ante contingencias. Se describen aspectos de interés para la determinación de flujos máximos de energía eléctrica a través de líneas de transmisión de redes eléctricas. Se establece el límite térmico de conductores eléctricos, la cargabilidad de líneas de transmisión y el límite de estabilidad ante oscilaciones interárea para un enlace del Sistema Interconectado Nacional (SIN) de México. Para el caso de estabilidad debido a oscilaciones interárea, se muestra la aplicación de un esquema de corte de carga para estabilizar un modo de oscilación interárea (Castellanos et al., 2008) al amortiguar oscilaciones negativas llevando con ello al sistema eléctrico a una condición de operación estable.

Los conductores eléctricos indicados en la determinación de los límites de transferencia son los que utiliza Comisión Federal de Electricidad (1996) (empresa eléctrica de México) en la red eléctrica de transmisión. Para evaluar el comportamiento dinámico de la red eléctrica se realizan simulaciones utilizando modelos y herramientas de análisis tanto lineal (Powertech Labs Ing., 2007a) como no lineal (Powertech Labs Ing., 2007b) de sistemas eléctricos de potencia.

\section{Límite térmico}

El límite térmico de un conductor para líneas aéreas es la corriente máxima permitida, considerando una temperatura máxima a través del conductor para condiciones ambientales establecidas. El cálculo del límite térmico para líneas aéreas se obtiene mediante métodos de balance de calor. Para determinar el límite térmico de conductores aéreos desnudos es necesario considerar en el análisis el efecto de la temperatura ambiente, la velocidad y dirección del viento, la emisión solar y la altura sobre el nivel del mar (IEEE Std. 738-2002).

En la actualidad, existen métodos tanto estáticos como dinámicos para determinar el límite térmico, la diferencia es que en el caso de los dinámicos algunas de las variables utilizadas en el cálculo se obtienen mediante mediciones en tiempo real (tensión, flecha, temperatura, corriente en el conductor) que se envían para ser procesadas en un centro de control.

Los conductores tipo ACSR (Aluminum Conductor Steel Reinforced) son los más utilizados a nivel mundial, estos conductores están diseñados para operar a una temperatura continua máxima de $100^{\circ} \mathrm{C}$, sin embargo, normalmente se supone una temperatura total de $75^{\circ} \mathrm{C}$ debido a que su uso tiene un buen desempeño del conductor (Westinghouse Electric Corporation, 1950). En general, las empresas eléctricas consideran en el análisis temperaturas de operación del conductor que van desde $50^{\circ} \mathrm{C}$ hasta $100^{\circ} \mathrm{C}$. En la figura 1 se muestra el comportamiento de la corriente a través de un conductor Bluejay, calibre 1113 KCM considerando las temperaturas de operación del conductor de $50^{\circ} \mathrm{C}$, $75^{\circ} \mathrm{C}$ y $100^{\circ} \mathrm{C}$ ante variaciones de la temperatura ambiente, velocidad del viento, ángulo del viento y altura sobre nivel del mar. A menos que se indique lo contrario, los valores considerados en el análisis son los siguientes: temperatura ambiente de $40^{\circ} \mathrm{C}$, velocidad del viento de $2 \mathrm{~m} / \mathrm{s}$, ángulo del viento $45^{\circ} \mathrm{C}$ (con respecto al conductor) y una elevación de $1600 \mathrm{~m}$ sobre el nivel del mar.

En la figura 1 se observa que al incrementar la capacidad de transferencia de corriente a través del conductor aumenta la temperatura del mismo, aquí se observa que para un mismo conductor pueden existir diferentes límites térmicos operativos basados en diferentes políticas operativas de las empresas eléctricas o del ingeniero de diseño. Por ejemplo, si una empresa eléctrica define que el conductor operará a una temperatura máxima de $75^{\circ} \mathrm{C}$ tendrá como restricción un límite térmico menor al obtenido por una empresa que considera operar el conductor a una temperatura máxima de $100^{\circ} \mathrm{C}$. Así, para una temperatura ambiente de $30^{\circ} \mathrm{C}$ la corriente a través del conductor tomará los valores de 919 A, 1317 A y 1575 A para temperaturas de operación de 50,75 y $100^{\circ} \mathrm{C}$, respectivamente, de manera que existe una diferencia de $656 \mathrm{~A}$ entre las temperaturas de $50^{\circ} \mathrm{C}$ y $100^{\circ} \mathrm{C}$. 
a)

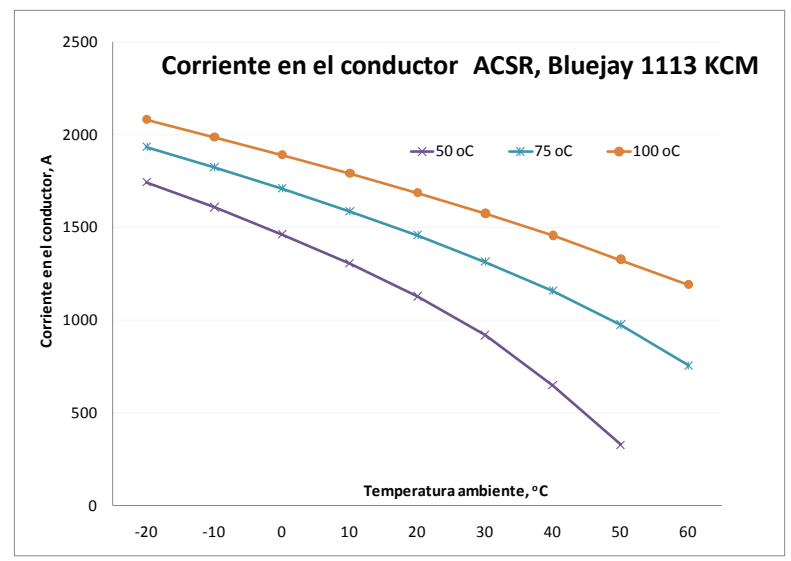

b)

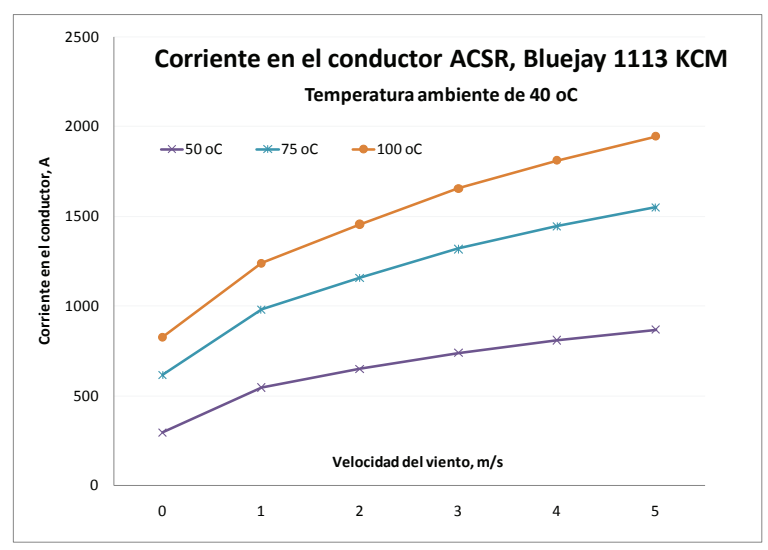

c)

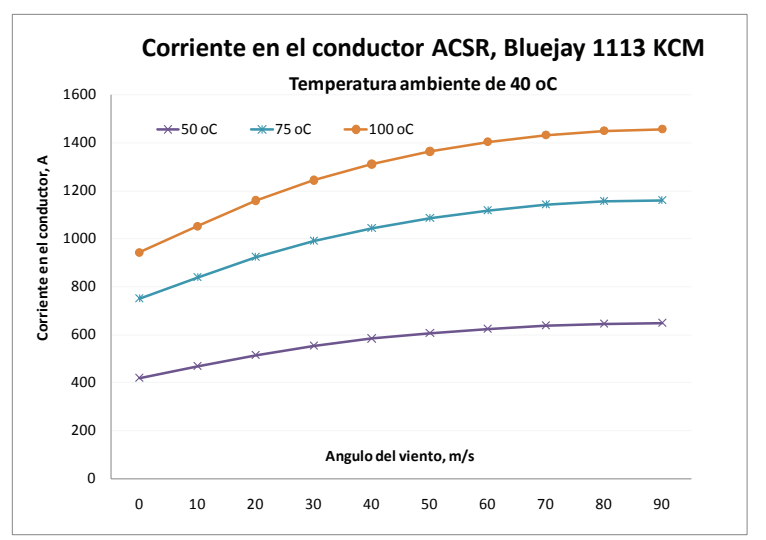

Figura 1. Efecto en la corriente del conductor Bluejay 1113 para temperaturas de operación de 50,75 y $100^{\circ} \mathrm{C}$ ante variaciones de las condiciones ambientales (climatológicas), a) corriente en el conductor Bluejay 1113 ante variación de la temperatura ambiente, b) corriente en el conductor Bluejay 1113 ante variación de la velocidad del viento, c) efecto en la corriente del conductor Bluejay 1113 ante variaciones de la dirección del viento
En la figura 1a, se observa que al incrementarse la temperatura ambiente disminuye la capacidad para transmitir corriente por el conductor. Así, considerando un incremento de temperatura ambiente de $0^{\circ} \mathrm{C}$ a $50^{\circ} \mathrm{C}$, la capacidad del conductor para transmitir corriente disminuye de 1711 a 978 A (733 A).

En la figura $1 b$, se observa que al incrementarse la velocidad del viento aumenta la capacidad para transmitir corriente por el conductor. Por ejemplo, considerando un incremento de la velocidad de viento de 2 a 4 $\mathrm{m} / \mathrm{s}$, la capacidad del conductor para transmitir corriente aumenta $285 \mathrm{~A}$ (de 1159 a 1444 A).

En la figura 1c, se muestra cómo al aumentar el ángulo del viento aumenta la capacidad para transmitir corriente por el conductor. Si el viento fluye de manera paralela al conductor, la capacidad de transferencia de corriente de este será mínima y si el viento corre perpendicular al conductor se obtiene el mayor nivel de transferencia de corriente.

En la figura 2, se presenta la curva característica corriente-temperatura de los conductores ACSR Bluejay, calibre 1113 KCM y Canary 900 KCM. Al aumentar la capacidad de transmisión de corriente se incrementa la temperatura de operación del conductor y entre mayor es la temperatura en el conductor mayor es la diferencia entre las capacidades de conducción de corriente de ambos calibres.

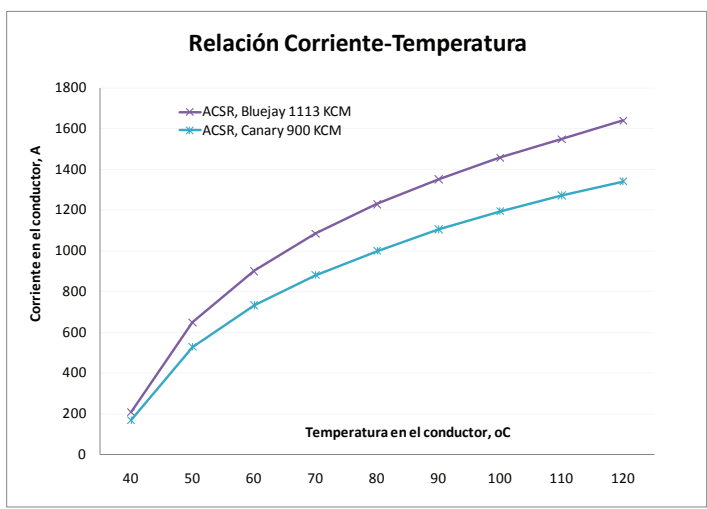

Figura 2. Efecto en la corriente de los conductores ACSR Bluejay 1113 y Canary 900 ante cambios en la temperatura de operación del conductor

Para incrementar el rango térmico de una línea (Cigre, 2007) es posible utilizar conductores que operan a una temperatura mayor, los cuales cumplen (en algunos casos mejoran) las restricciones de flecha y tensión de la línea. Estos conductores conocidos como de alta temperatura pueden operar a temperaturas y capacidades de transferencia de hasta 100\% más que la de los conductores convencionales (ACSR). 
En la figura 3 se observa la curva característica corriente-temperatura para el conductor de alta temperatura tipo ACCS Bluejay 1113 KCM. Este conductor está diseñado para operar a temperaturas de hasta $180^{\circ} \mathrm{C}$, lo que le da la capacidad para transmitir una mayor cantidad de corriente que un conductor convencional (ACSR) de calibre similar.

Una ventaja que ofrecen los conductores de alta temperatura es que se pueden instalar en las mismas torres del derecho de vía existente, sin necesidad de reforzarlas. Respecto a los herrajes y accesorios de la línea, estos se reemplazan en función del tipo de conductor de alta temperatura a utilizar (Cigre, 2007).

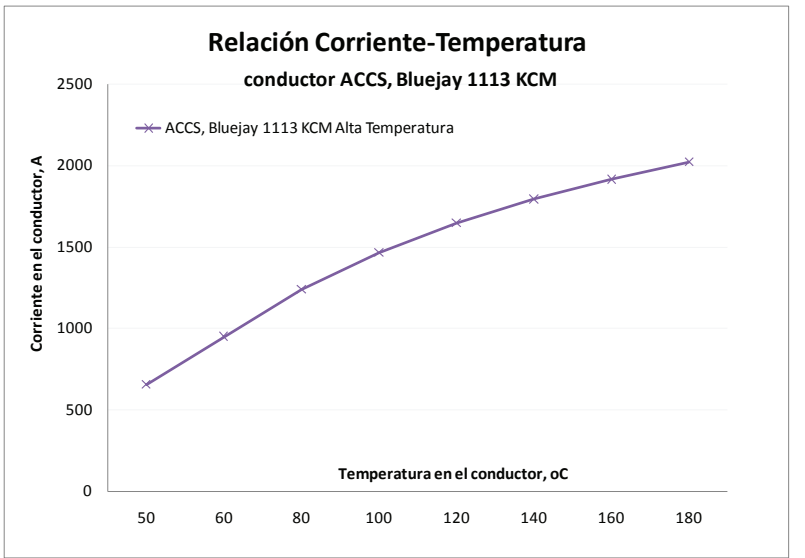

Figura 3. Efecto en la corriente del conductor de alta temperatura tipo ACCS, Bluejay 1113 ante variaciones de su temperatura de operación

Como se muestra en los párrafos anteriores, la determinación del límite térmico de conductores depende de una gran cantidad de criterios, los cuales están definidos de acuerdo con los criterios de diseño de las empresas eléctricas. Al considerar criterios con valores muy conservadores, los enlaces trasmiten niveles de energía menores al límite real, por el contrario, seleccionando parámetros más restrictivos los enlaces podrían violar sus niveles máximos de transferencia de energía.

Para contrarrestar las restricciones en la determinación del límite térmico indicadas en el párrafo anterior y, con ello, aprovechar al máximo la capacidad de transferencia de un enlace o línea eléctrica se puede utilizar la metodología en la que el límite térmico se obtiene de forma dinámica (Kyeon et al., 2001). Esto se logra a través de la medición de las condiciones ambientales y parámetros de la línea eléctrica: flujo de corriente, flecha y libramiento entre el conductor y tierra. Estos métodos permiten conocer la capacidad del enlace en tiempo real, lo que lleva a poder transmitir el nivel máximo de energía eléctrica de manera segura.
Las líneas de energía eléctrica de longitud pequeña (Duncan, 1990) son las que podrían llegar a transmitir niveles de potencia cercanos a su límite térmico. Para el caso de líneas de longitud media o alta existen restricciones que impiden que estas puedan operarse, dentro de márgenes de seguridad adecuados, con valores de transferencia de MW cercanos a su límite térmico.

Algunas restricciones que limitan la operación de líneas eléctricas a niveles de transferencia de potencia menores al límite térmico se analizan en los siguientes incisos.

\section{Límite por cargabilidad}

La cargabilidad de una línea de transmisión indica la capacidad de potencia que puede fluir por la línea bajo condiciones de operación aceptables. La cargabilidad de la línea está en función del calibre y la longitud de la línea, para conocer su comportamiento se consideran condiciones óptimas de voltaje en ambos extremos de la misma, es decir, un voltaje de 1 p.u. tanto en el nodo de envío como en el de recepción, como se muestra en la figura 4.

La cargabilidad de una línea puede valorarse a partir de la relación del flujo de potencia real contra potencia reactiva $(\mathrm{P}-\mathrm{Q})$ a través de la línea eléctrica. Esta curva característica (P-Q) varía tanto por el flujo de potencia como por la longitud de la línea. La curva característica potencia real-potencia reactiva de una línea eléctrica indica que al incrementar su flujo de potencia real, aumenta la potencia reactiva que esta consume, lo que se manifiesta como pérdida de potencia en la reactancia inductiva en serie de la línea. El valor de la potencia real donde el requerimiento de potencia reactiva es cero se conoce como SIL Surge Impedance Loading o potencia natural de la línea (Kundur, 1994; Duncan, 1990). El valor del SIL depende tanto de la inductancia serie y la capacitancia en derivación como del nivel de tensión entre fases de la línea de transmisión, como se indica en la ecuación 1. Cuanto mayor es el nivel de tensión mayor es el SIL de la línea. De igual manera, el SIL aumenta al incrementar el número de conductores por fase de la línea, ya que, disminuye su impedancia característica $\left[Z_{c}=\sqrt{(L / C)}\right]$

$S I L=\frac{K V_{L-L}^{2}}{\sqrt{\frac{L}{C}}}$

El comportamiento de la curva potencia real- potencia reactiva de la línea de transmisión se puede describir como sigue: 


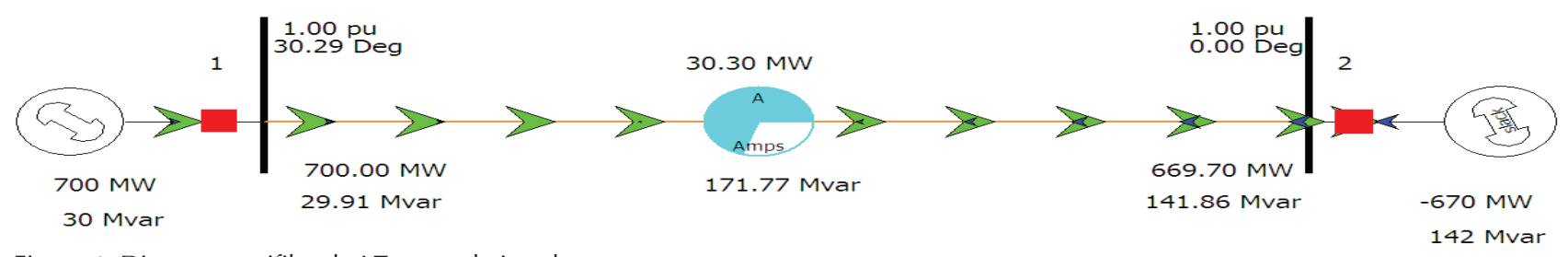

Figura 4. Diagrama unifilar de LT con voltajes planos

- Para niveles de transferencia de potencia real menores al SIL, la potencia capacitiva producida por la línea es menor a la potencia inductiva que esta consume, bajo estas condiciones la línea aporta potencia reactiva capacitiva a la red eléctrica.

- Cuando el flujo de potencia real a través de la línea es igual al valor de su SIL la potencia capacitiva producida por la línea es igual a la potencia inductiva que esta consume. Bajo esta condición de operación, la línea no inyecta, pero tampoco absorbe potencia reactiva de la red eléctrica.

- Finalmente, al aumentar el flujo de potencia real por la línea a valores superiores al SIL la potencia reactiva consumida por la línea es mayor a la potencia reactiva generada por la línea eléctrica, en este caso la línea demanda potencia reactiva de tipo inductiva que debe ser suministrada por otras fuentes del sistema eléctrico de potencia.

En la figura 5, se muestran curvas características de la relación de potencia real-potencias reactiva de una línea eléctrica de $230 \mathrm{kV}$ con un circuito, conductor ACSR, Bluejay, calibre $1113 \mathrm{KCM}$ y un conductor por fase para longitudes de 50, 100, 200 y $300 \mathrm{~km}$. Se observa como al incrementar el flujo de potencia real (MW) a través de la línea, se incrementa el consumo de potencia reactiva (MVARs) en la reactancia inductiva serie de la línea eléctrica. En este caso, el SIL de la línea de transmisión es de 143 MW. Se puede observar, que el SIL de la línea es independiente de su longitud.

La diferencia de los ángulos de voltaje entre ambos extremos de la línea es un parámetro importante que se recomienda mantener dentro de valores cercanos a 35 grados para operar el sistema eléctrico en condiciones de operación estables, evitando con ello, que ante una contingencia esta diferencia angular se incremente por arriba del límite máximo de estabilidad de estado estable (90 grados) lo que podría llevar al sistema eléctrico a una condición de inestabilidad.

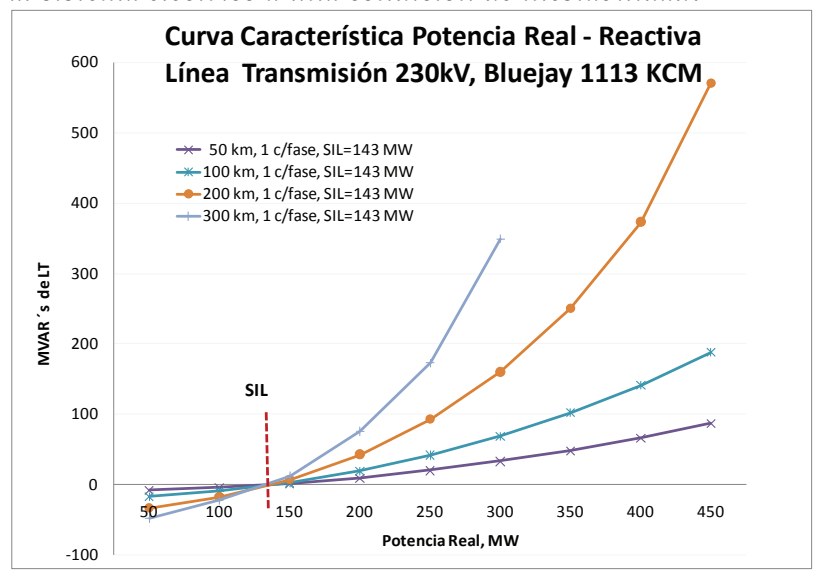

Figura 5. Curva característica potencia real-potencias reactiva para varias longitudes de una línea de $230 \mathrm{kV}$, un circuito, cable ACSR, Bluejay 1113 KCM con un conductor por fase

En la figura 6 se muestra la diferencia entre los ángulos de voltaje de los extremos de una línea de $230 \mathrm{kV}$, un circuito, cable ACSR, Bluejay, calibe $1113 \mathrm{KCM}$ con un conductor por fase para longitudes de 100, 200 y 300 $\mathrm{km}$. Se puede observar que al incrementar el flujo de potencia real por la línea, aumenta la separación angular entre ambos extremos de la línea. Considerando una separación angular máxima de 35 grados, el límite de estabilidad de estado estable de esta línea es $200 \mathrm{MW}$ para una longitud de $300 \mathrm{~km}, 300 \mathrm{MW}$ para una longitud de $200 \mathrm{~km}$ y $600 \mathrm{MW}$ para una longitud de $100 \mathrm{~km}$ (no mostrado en la figura). También, se observa que cuanto mayor sea el nivel de transferencia de potencia real mayor será la separación angular entre los extremos de la línea de distintas longitudes.

En la figura 7 se muestran curvas características de la relación de potencia real-potencias reactiva de una línea eléctrica de $400 \mathrm{kV}$ con un circuito, conductor 


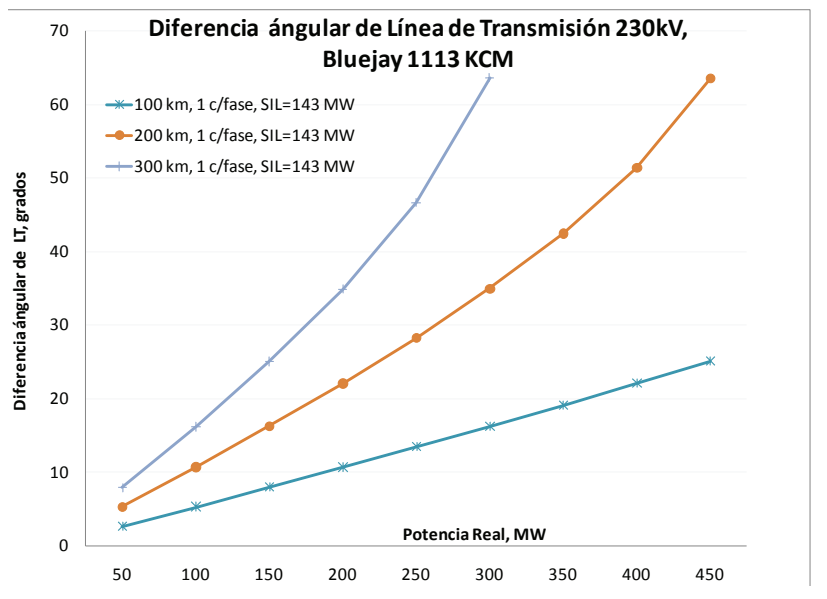

Figura 6. Diferencia angular para varias longitudes de una línea de $230 \mathrm{kV}$, un circuito, cable ACSR, Bluejay 1113 KCM con un conductor por fase

ACSR, Bluejay, calibre $1113 \mathrm{KCM}$ y dos conductores por fase para longitudes de 100, 200 y $300 \mathrm{~km}$. Se observa cómo al incrementar el flujo de potencia real (MW) a través de la línea, se incrementa el consumo de potencia reactiva (MVAR) en la reactancia inductiva serie de la línea eléctrica. En este caso, el SIL de la línea de transmisión es 522 MW. Se puede observar, que igual que para el caso de $230 \mathrm{kV}$ (figura 5), el SIL de la línea es independiente de su longitud.

De la curva característica de potencia real-potencias reactiva de una línea eléctrica de $100 \mathrm{~km}$, un circuito, cable ACSR, Bluejay, calibre 1113 KCM con un conductor por fase, mostradas en las figuras 5 y 7 , se observa un incremento de $250 \mathrm{MW}$ en el valor del SIL al operar la línea en $400 \mathrm{kV}$ respecto a operarla en $230 \mathrm{kV}$.

En la figura 8 se muestran curvas características de la relación de potencia real-potencia reactiva de una línea eléctrica de $400 \mathrm{kV}$, un circuito, conductor ACSR, Bluejay, calibre $1113 \mathrm{KCM}, 100 \mathrm{~km}$ con uno, dos y tres conductores por fase, respectivamente. Se observa cómo al incrementar el número de conductores por fase se incrementa la potencia reactiva capacitiva suministrada por la línea y se reduce la potencia reactancia inductiva absorbida por la línea eléctrica. En este caso, cuando la línea de transmisión tiene uno, dos y tres conductores por fase el SIL es 393, 522 y 585 MW, respectivamente.

En la figura 9 se muestra la diferencia entre los ángulos de voltaje de los extremos de una línea de $400 \mathrm{kV}$, un circuito, cable ACSR, Bluejay, calibe $1113 \mathrm{KCM}, 100$ $\mathrm{km}$ de longitud con uno, dos y tres conductores por fase. Se puede observar que al incrementar el flujo de potencia real por la línea, aumenta la separación angular entre ambos extremos de la línea. Se observa que cuanto mayor es el número de conductores por fase

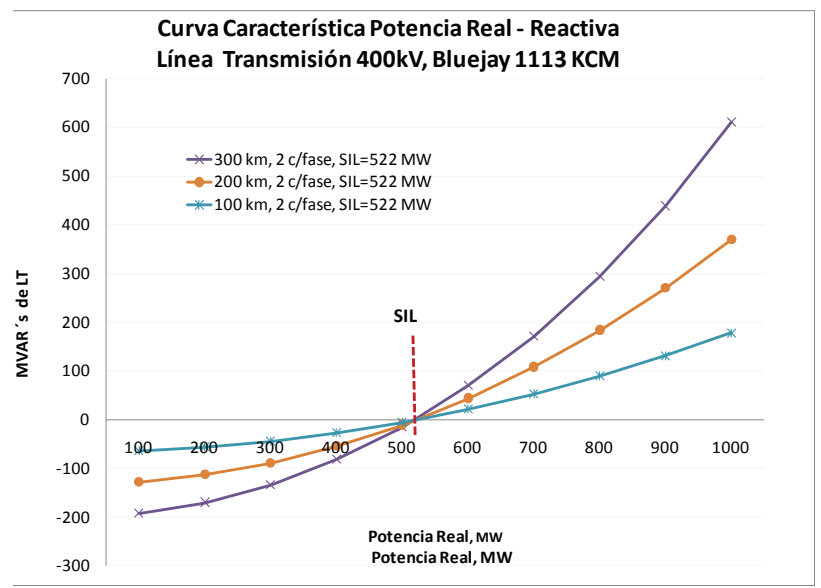

Figura 7. Curva característica potencia real-potencias reactiva para varias longitudes de una línea de $400 \mathrm{kV}$, un circuito, cable ACSR Bluejay 1113 KCM con dos conductores por fase

menor es la separación angular del voltaje en ambos extremos de la línea. La separación angular entre los voltajes de los extremos de la línea es menor al incrementar el nivel de tensión.

En la figura 10 se puede observar que la separación angular entre el voltaje de ambos extremos es mayor cuando la longitud de la línea eléctrica es mayor.

En la figura 11 se observa el comportamiento de las pérdidas de potencia real de una línea de $400 \mathrm{kV}$, un circuito, cable ACSR, Bluejay, calibe 1113 KCM para diferente longitud de línea (figura 11a) y para distinto número de conductores por fase (figura 11b). Las pérdidas de MW presentan un comportamiento cuadrático ( $\left.I^{2} R\right)$. Como se observa en la figura 11a, las pérdidas de potencia real se incrementan al aumentar el flujo de potencia real y la longitud de la línea. Por el contrario, las pérdidas de MW disminuyen al aumentar el número de conductores por fase.

\section{Límite por estabilidad ante oscilaciones interárea}

El problema de oscilaciones de potencia de tipo interárea (Kundur, 1994) se puede presentar al debilitar los enlaces de transmisión entre distintas regiones geográficas. Los enlaces débiles se caracterizan por tener niveles elevados de transferencia de potencia o altas impedancias. Por lo que, una relación de amortiguamiento aceptable se puede obtener disminuyendo el flujo de potencia o reduciendo la impedancia equivalente del enlace de transmisión asociado al modo de oscilación interárea. Para reducir el flujo por el enlace se puede desconectar carga del lado de recepción o desconectar la generación del lado de envío, buscando resolver el déficit de suministro desde otras fuentes. 


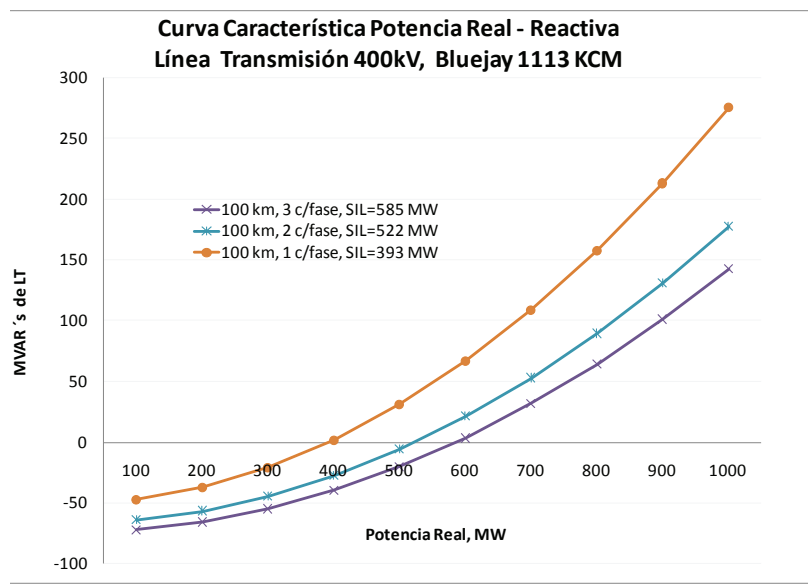

Figura 8. Curva característica potencia real-potencias reactiva para una línea de $400 \mathrm{kV}$, un circuito, cable ACSR, Bluejay 1113 $\mathrm{KCM}$ con uno, dos y tres conductores por fase, $100 \mathrm{~km}$

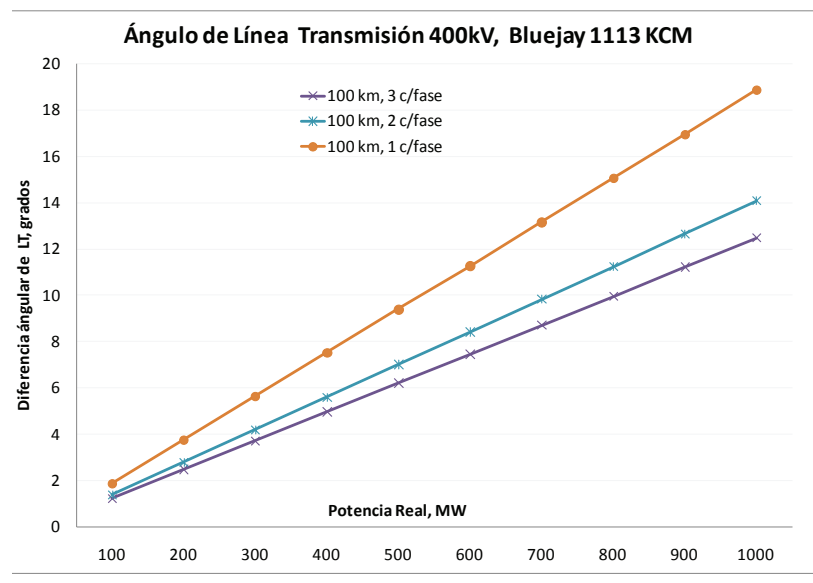

Figura 9. Curva característica potencia real-separación angular para una línea de $400 \mathrm{kV}$, un circuito, cable ACSR, Bluejay 1113 $\mathrm{KCM}$ con uno, dos y tres conductores por fase, $100 \mathrm{~km}$

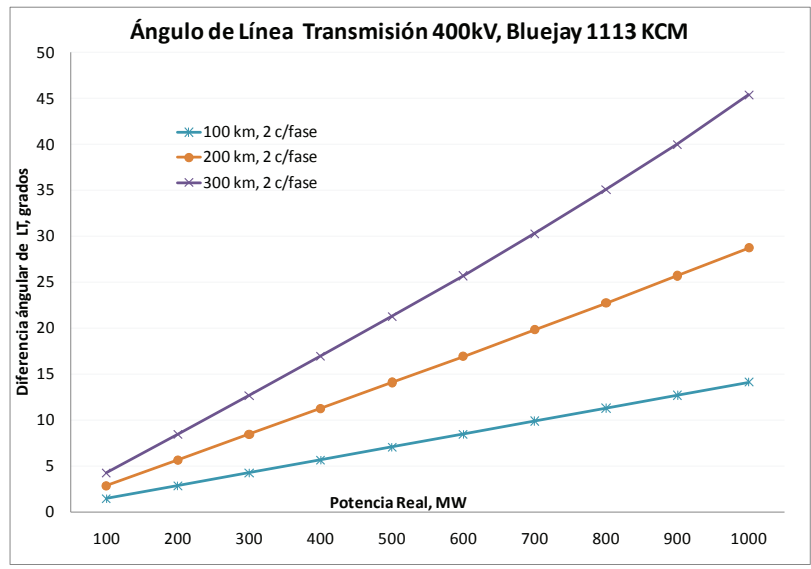

Figura 10. Curva característica potencia real-potencias reactiva para varias longitudes de una línea de $400 \mathrm{kV}$, un circuito, cable ACSR, Bluejay 1113 KCM con dos conductores por fase
Respecto a la impedancia equivalente, esta se puede reducir aumentando el número de circuitos del enlace.

En el diagrama de flujo de la figura 12, se presenta un algoritmo para determinar el límite de transferencias de un enlace ante oscilaciones de tipo interárea.

El algoritmo mostrado en la figura 12 se describe a continuación:

- Caracterización del modo de oscilación. El proceso de caracterización del modo de oscilación consiste en determinar los grupos de máquinas que intervienen con mayor participación en el modo de oscilación bajo estudio, para ello podemos utilizar el análisis de valores propios, análisis modal y factores de participación. Además, esta información es importante para ubicar el enlace crítico asociado al modo de oscilación interárea.

En el Sistema Interconectado Mexicano se pueden excitar varios modos de oscilación tanto del tipo local como interárea (Castellanos et al., 2007 y 2006), vea la figura 13. El modo interárea llamado Norte-Sur de $0.32 \mathrm{~Hz}$ involucra la acción de máquinas del Norte oscilando en oposición con máquinas de los sistemas del Sur de la red eléctrica. El modo peninsular es de $0.51 \mathrm{~Hz}$ y se caracteriza por la oscilación entre unidades de la Península de Yucatán contra máquinas del Área Occidental. El tercer modo mostrado en la figura es de $0.76 \mathrm{~Hz}$ y su dinámica se debe a la oscilación entre máquinas síncronas de del Área Oriental contra unidades del Área Occidental.

Este artículo se enfoca en determinar el límite de estabilidad ante disturbios pequeños del enlace HuinalaTamos ubicado entre las áreas del Norte y del Sur del SIN; este enlace está constituido por una línea de 400 $\mathrm{kV}$ con doble circuito y dos conductores por fase en cada uno de ellos. También, se revisa el comportamiento dinámico del enlace entre las áreas Peninsular-Oriental, el cual consta de tres circuitos de $230 \mathrm{kV}$.

- Identificar contingencias críticas. Las contingencias críticas son aquellas que provocan una oscilación con amortiguamiento cero o negativo, ya que estas llevan al sistema eléctrico a una condición de inestabilidad.

Es recomendable realizar un barrido de contingencias tanto sencillas como múltiples para detectar aquellas que llevan la red eléctrica a una condición de operación inestable. El análisis de contingencias se puede realizar en el dominio de la frecuencia (estabilidad ante disturbios pequeños) y en el dominio del tiempo (estabilidad 
a)

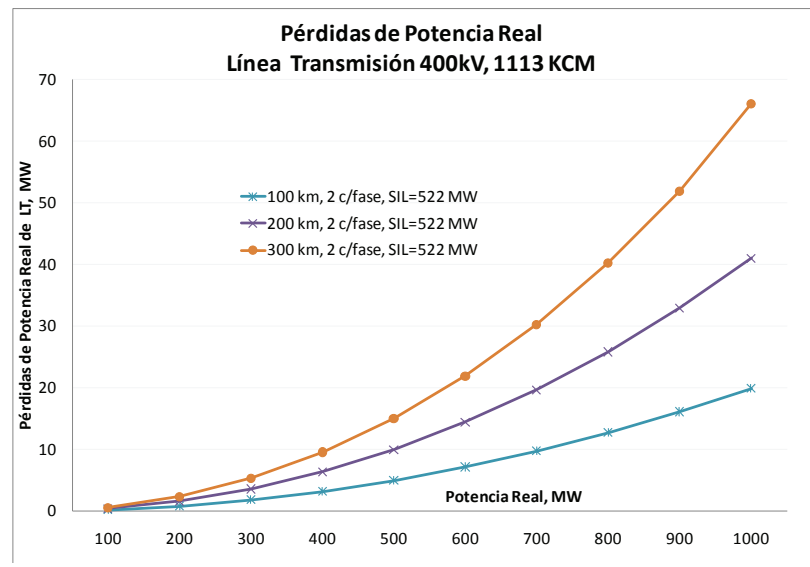

b)

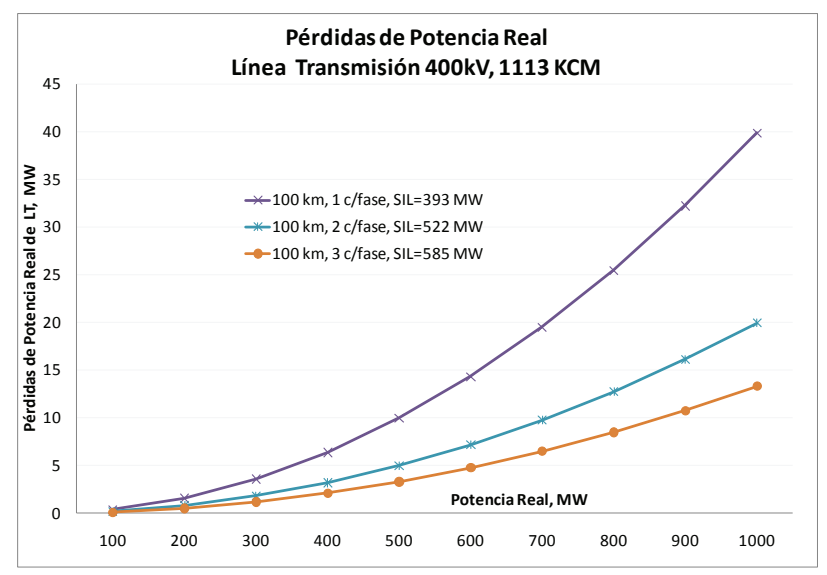

Figura 11. Curva característica potencia real-separación angular de una línea de 400kVcable ACSR, Bluejay 1113 KCM, un circuito, a) 100, 200 y $300 \mathrm{~km}$ de longitud con dos conductor por fase, b) $100 \mathrm{~km}$ de longitud con uno, dos y tres conductor por fase

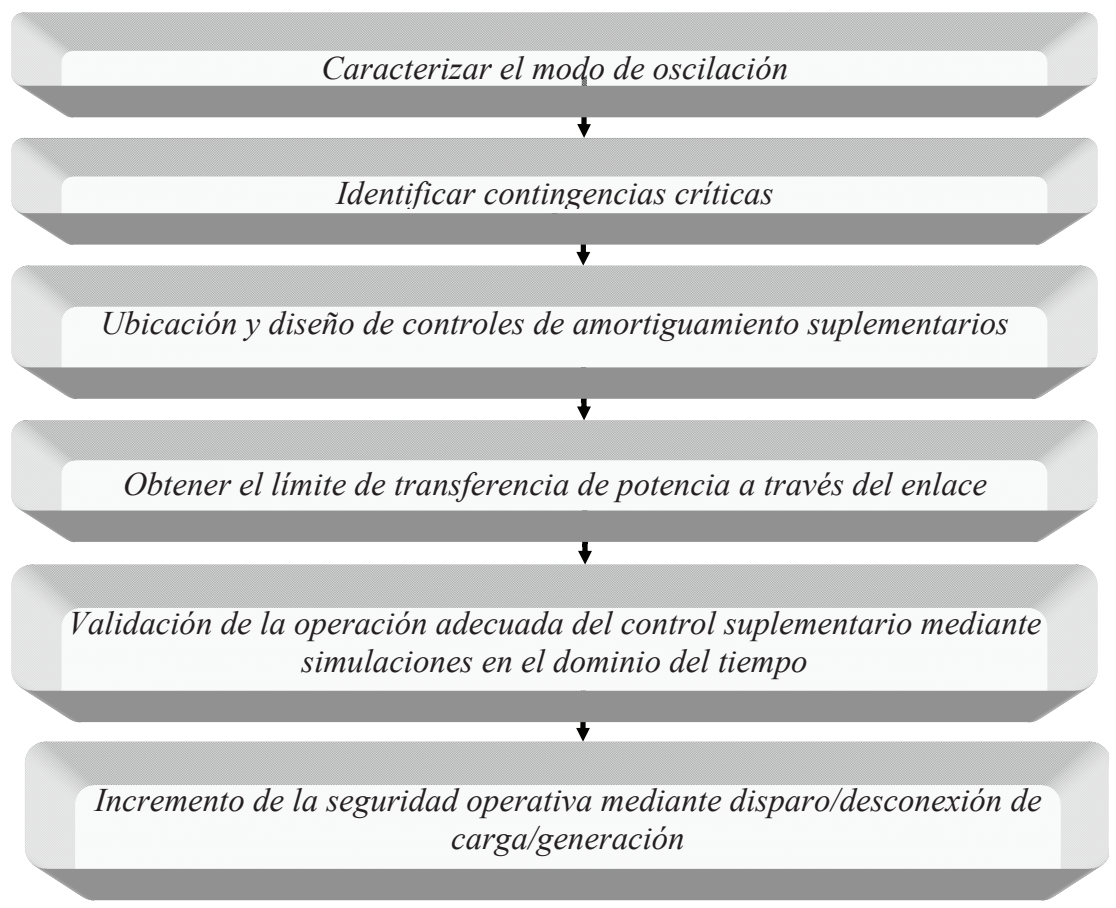

Figura 12. Determinación de límite de estabilidad angular de enlaces de transmisión transitoria), este último permite considerar en el análisis diversos tipos de fallas que dan información de la severidad asociada a la contingencia.

Por un lado en los casos 8 y 10 de la tabla 1, se muestran resultados de la aplicación del análisis de contingencia en el dominio de la frecuencia, en este caso a través de la frecuencia de oscilación y relación de amortiguamiento del modo se valora la severidad de la contingencia. Por otro lado en la figura 14, se presentan resultados de simulaciones de contingencias en el dominio del tiempo mostrando en este caso, el comportamiento dinámico de tres contingencias críticas sencillas que provocan oscilaciones con amortiguamiento negativo (inestabilidad) del modo interárea Norte-Sur, $0.32 \mathrm{HZ}$. Las contingencias consideradas son: $i$ ) el disparo sin falla de la unidad 1 de la planta de Laguna Verde, ii) falla trifásica en la línea Laj_Gue_400 kV, disparando el circuito uno al momento de liberar la falla, y iii) falla trifásica en el Bus Gue_400 $\mathrm{kV}$, disparando el circuito uno de la línea Laj_Gue-400 $\mathrm{kV}$ al momento de liberar la falla. 


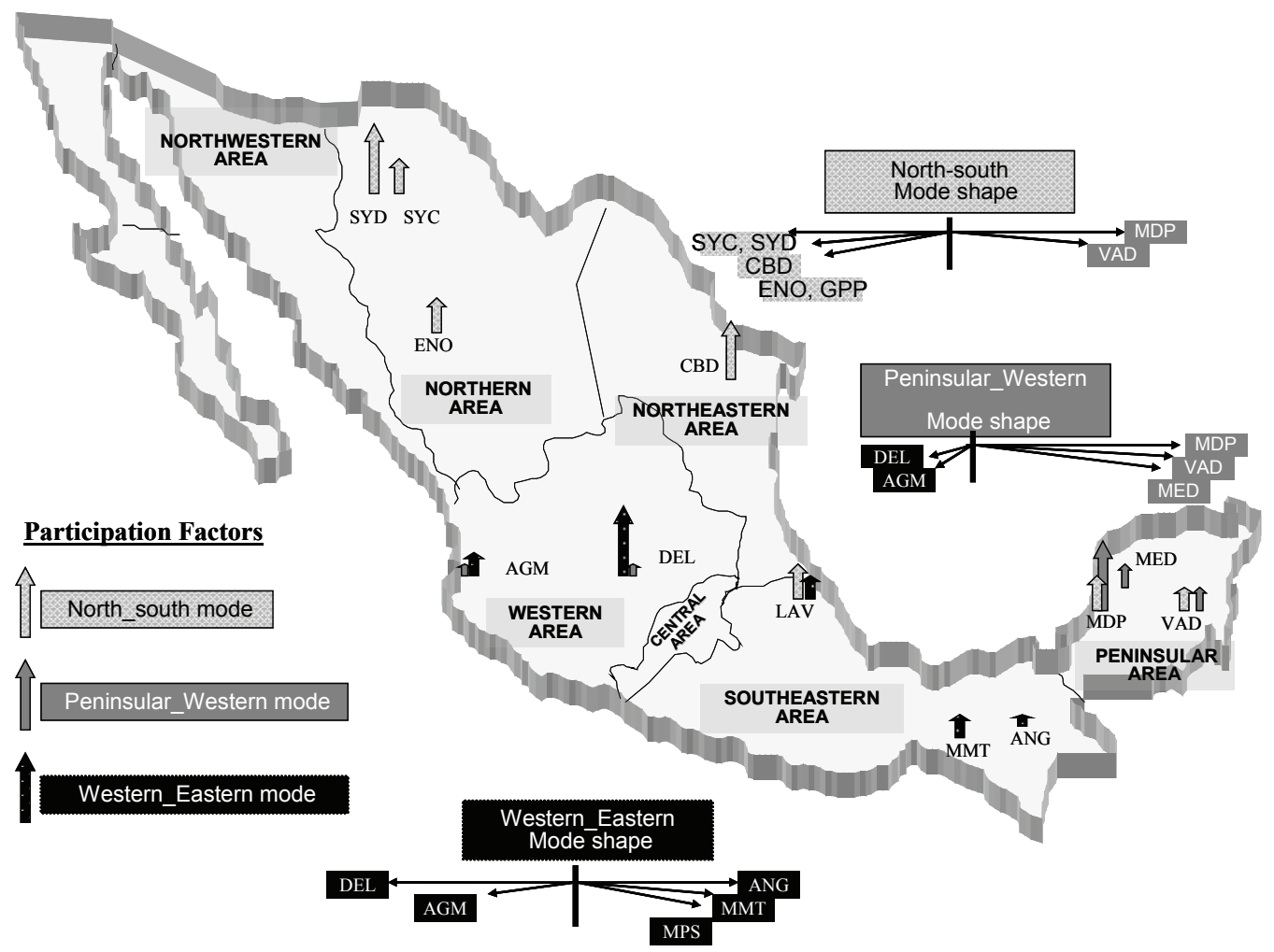

Figura 13. Visualización de modos de oscilación interárea en el Sistema Interconectado Mexicano

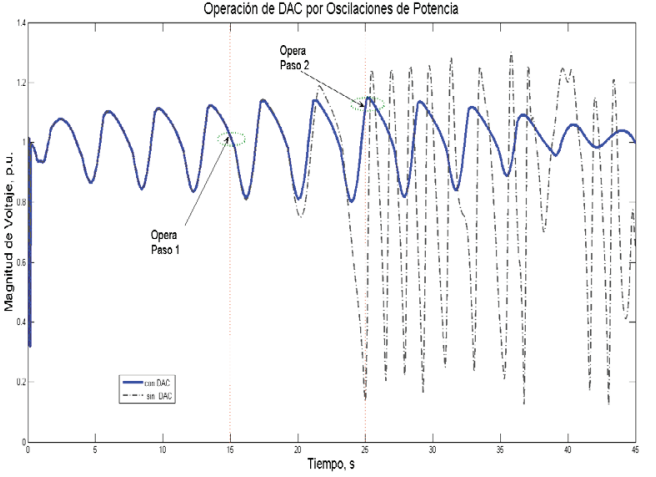

Figura 14. Identificación de contingencias críticas modo de oscilación interárea Norte-Sur

- Ubicación y diseño de controles de amortiguamiento suplementarios. Las máquinas óptimas para la ubicación de estabilizadores del sistema de potencia (PSS, Power System Stabilizer) (controles de amortiguamiento suplementarios) son aquellas que tienen un alto factor de participación y alta contribución en la composición del modo de oscilación.
Las máquinas síncronas de las plantas de Samalayuca (SYU, SYD) y Carbón Dos (CBD) presentan altos factores de participación y alta actividad en la composición del modo de oscilación interárea de $0.32 \mathrm{~Hz}$. Respecto al modo de oscilación interárea Peninsular $(0.52 \mathrm{~Hz})$, la unidades que tienen altos factores de participación y alta actividad en el modo de oscilación son las de la planta Merida Potencia Dos (MDP). Lo anterior indica que agregando estabilizadores del sistema de potencia en unidades se mejora el amortiguamiento del modo de oscilación. Para determinar los parámetros de los PSS (ganancia, constantes de tiempo de los bloques de adelanto-atraso, señal de entrada) se puede utilizar la metodología indicada en Kundur (1994) y Castellanos et al. (2006).

- Límite de transferencia de potencia. Este límite está definido por el monto del flujo de potencia real a través del enlace para el cual la relación de amortiguamiento del modo interárea es mayor o igual a 3\%, tanto para condiciones de operación de precontingencia como ante contingencias. Este índice de la relación amortiguamiento mínimo de 3\% es un valor recomendado (System Oscillation Working Group, 1995; CIGRE, 1996), que puede variar en 
función de las características y políticas operación de cada red eléctrica.

En la tabla 1 se muestran los parámetros de los modos de oscilación Norte-Sur y Peninsular-Occidental para diversos flujos por los enlaces entre áreas y considerando diversos estabilizadores del sistema de potencia (PSS) tanto para condiciones de operación en precontingencia como ante contingencia. La contingencia considerada es la desconexión de la línea Laj_Gue-400 kV. En el Sistema Eléctrico Interconectado Mexicano hay otros PSS, sin embargo aquí solo se indican aquellos que tienen una fuerte influencia en el comportamiento de los modos interárea bajo estudio.

De los resultados mostrados en la tabla 1 se observa lo siguiente:

- Al incrementar el flujo de potencia por el enlace asociado al modo se reduce tanto la relación de amortiguamiento como la frecuencia de la oscilación. Por ejemplo, al comparar el caso 1 con el caso 2 se observa como al incrementar el flujo de potencia del enlace Norte-Sur de 800 a 935 MW la relación de amortiguamiento del modo Norte-Sur se reduce de 5.6 a $3.86 \%$. En el caso del modo peninsular, al ob- servar los casos 2 y 3 se ve que al incrementar el flujo de potencia del enlace Peninsular-Oriental de 110 a 390 MW el modo se hace inestable al reducirse la relación de amortiguamiento de 4.21 a $-3.66 \%$.

- Al incorporar más estabilizadores del sistema de potencia, con alta participación, se incrementa la relación de amortiguamiento del modo de oscilación, es decir, el sistema eléctrico aumenta su margen de estabilidad angular. Por ejemplo, si comparamos el caso 3 con el caso 7 se observa que la relación de amortiguamiento del modo Norte-Sur se incrementa de 3.03 a $18.3 \%$ al agregar los PSS en otras tres unidades síncronas (CBD U4 y SYU U1-U2); por su parte, el modo Peninsular incrementa su relación de amortiguamiento de $\mathbf{- 3 . 6 6}$ a $11.85 \%$ al incorporar PSS en la unidades 1 y 2 de MDP.

- Ante contingencias críticas se reduce la relación de amortiguamiento como la frecuencia de la oscilación. Por ejemplo, comparando los casos 7 y 8, así como los casos 9 y 10, podemos observar que ante la salida de la desconexión de un circuito de la línea Laj-Gue_400 $\mathrm{kV}$ la relación de amortiguamiento disminuye, de especial importancia es el caso 10 donde se presenta una inestabilidad $(\zeta=-\mathbf{0 . 6 7})$ del modo de oscilación.

\begin{tabular}{|c|c|c|c|c|}
\hline Caso & Flujo por enlace, MW & $\begin{array}{c}\text { Ubicación de } \\
\text { los PSS }\end{array}$ & $\begin{array}{c}\text { Modo Norte- } \\
\text { Sur } \\
\lambda^{*} \\
(\zeta, \mathrm{f})^{* *}\end{array}$ & $\begin{array}{c}\text { Modo } \\
\text { Peninsular } \\
\lambda^{*} \\
(\zeta, \mathrm{f})^{* *}\end{array}$ \\
\hline 1 & $\begin{array}{lr}\text { NorteSur } & 800 \\
\text { Peninsular } & 110\end{array}$ & $\begin{array}{l}\text { CBD U1-U3 } \\
\text { MDP U3 }\end{array}$ & $\begin{array}{c}-0.114 \pm j 2.04 \\
(0.325,5.6)\end{array}$ & $\begin{array}{r}-0.138 \pm j 3.27 \\
(0.52,4.20)\end{array}$ \\
\hline 2 & $\begin{array}{lr}\text { NorteSur } & 935 \\
\text { Peninsular } & 110\end{array}$ & $\begin{array}{l}\text { CBD U1-U3 } \\
\text { MDP U3 }\end{array}$ & $\begin{array}{l}-0.076 \pm j 1.97 \\
(0.313,3.86)\end{array}$ & $\begin{array}{r}-0.138 \pm j 3.27 \\
(0.52,4.21)\end{array}$ \\
\hline 3 & $\begin{array}{lr}\text { NorteSur } & 935 \\
\text { Peninsular } & 390\end{array}$ & $\begin{array}{l}\text { CBD U1-U3 } \\
\text { MDP U3 }\end{array}$ & $\begin{array}{l}-0.059 \pm j 1.96 \\
(0.312,3.03)\end{array}$ & $\begin{array}{l}0.138 \pm j 3.27 \\
(0.47,-3.66)\end{array}$ \\
\hline 4 & $\begin{array}{lr}\text { NorteSur } & 800 \\
\text { Peninsular } & 390\end{array}$ & $\begin{array}{l}\text { CBD U1-U3 } \\
\text { MDP U3 }\end{array}$ & $\begin{array}{l}-0.094 \pm j 2.03 \\
(0.324,4.61)\end{array}$ & $\begin{array}{c}0.138 \pm 3.27 \\
(0.47,-3.53)\end{array}$ \\
\hline 5 & $\begin{array}{lr}\text { NorteSur } & 800 \\
\text { Peninsular } & 110 \\
\end{array}$ & $\begin{array}{l}\text { CBD U1-U4 } \\
\text { MDP U1-U3 }\end{array}$ & $\begin{array}{l}-0.182 \pm j 2.06 \\
(0.328,8.77)\end{array}$ & $\begin{array}{l}-0.600 \pm j 3.29 \\
(0.52,17.91)\end{array}$ \\
\hline 6 & $\begin{array}{lr}\text { NorteSur } & 935 \\
\text { Peninsular } & 390\end{array}$ & $\begin{array}{l}\text { CBD U1-U4 } \\
\text { MDP U1-U3 }\end{array}$ & $\begin{array}{c}-0.139 \pm j 1.95 \\
(0.311,7.1)\end{array}$ & $\begin{array}{l}-0.321 \pm j 2.83 \\
(0.45,11.26)\end{array}$ \\
\hline 7 & $\begin{array}{lr}\text { NorteSur } & 935 \\
\text { Peninsular } & 390\end{array}$ & $\begin{array}{l}\text { CBD U1-U4 } \\
\text { SYU U1-U2 } \\
\text { MDP U1-U3 }\end{array}$ & $\begin{array}{l}-0.371 \pm j 1.99 \\
(0.317,18.3)\end{array}$ & $\begin{array}{l}-0.340 \pm j 2.85 \\
(0.45,11.85)\end{array}$ \\
\hline 8 & $\begin{array}{cc}\text { NorteSur } & 935 \\
\text { Peninsular } & 390 \\
\text { Sin LAJ-GUE } 400 \text { kV, C2 }\end{array}$ & $\begin{array}{l}\text { CBD U1-U4 } \\
\text { SYU U1-U2 } \\
\text { MDP U1-U3 }\end{array}$ & $\begin{array}{l}-0.202 \pm j 1.77 \\
(0.282,11.3)\end{array}$ & $\begin{array}{c}-0.332 \pm j 2.81 \\
(0.448,11.72)\end{array}$ \\
\hline 9 & $\begin{array}{lr}\text { NorteSur } & 935 \\
\text { Peninsular } & 390\end{array}$ & $\begin{array}{l}\text { SYU U1-U2 } \\
\text { MDP U1-U3 }\end{array}$ & $\begin{array}{l}-0.140 \pm j 1.98 \\
(0.315,7.06)\end{array}$ & $\begin{array}{l}-0.328 \pm j 2.84 \\
(0.45,11.45)\end{array}$ \\
\hline 10 & $\begin{array}{cc}\text { NorteSur } & 935 \\
\text { Peninsular } & 390 \\
\text { Sin LAJ-GUE } 400 \text { kV, C2 }\end{array}$ & $\begin{array}{l}\text { SYU U1-U2 } \\
\text { MDP U1-U3 }\end{array}$ & $\begin{array}{c}0.012 \pm \mathrm{j} 1.76 \\
(0.28,-0.67)\end{array}$ & $\begin{array}{l}-0.329 \pm j 2.81 \\
(0.45,11.63)\end{array}$ \\
\hline
\end{tabular}

Tabla 1. Parámetros de modos de oscilación Norte-Sur y Peninsular-Oriental ante diversos flujos de potencia por enlaces y diversos PSSs

\footnotetext{
$* \lambda$ es el valor propio del modo de oscilación, en $1 / \mathrm{s}$ y rad.

**Relación de amortiguamiento $(\zeta)$ en \% y la frecuencia (f) en $\mathrm{Hz}$
} 
- El incremento en los flujos de potencia de un enlace asociado con un modo de oscilación (por ejemplo el Peninsular-Oriental, $0.52 \mathrm{~Hz}$ ) puede excitar otro modo de oscilación localizado en otras áreas regiones de la red eléctrica (por ejemplo, el modo NorteSur, $0.32 \mathrm{~Hz}$ ), esto se aprecia claramente en los resultados de las simulaciones realizadas utilizando el sistema no-lineal mostrado en el siguiente inciso.

De los resultados del análisis de valores propios se observa que el límite de estabilidad, ante disturbios pequeños del enlace está en función de una gran cantidad de variables como son: el nivel de flujo de potencia por el enlace, la impedancia equivalente del enlace, el número de máquinas síncronas con PSS.

- Validación de la operación adecuada del control suplementario mediante simulaciones en el dominio del tiempo. Para garantizar una operación adecuada de los controles de amortiguamiento, se realizan simulaciones utilizando el modelo no lineal del sistema eléctrico interconectado (análisis en el dominio del tiempo), como se muestra en las figuras 15 a 17. La contingencia simulada es el disparo sin falla de uno de los dos circuitos de la línea Laj_Gue_400 kV y se consideran los PSS en CBDU1-U3 y MDP_U3.

La figura 15 muestra el comportamiento dinámico del modo de oscilación interárea de $0.32 \mathrm{~Hz}$ ante el incremento del flujo de potencia de 810 a 935 MW a través del enlace Norte-Sur. Se observa que ante la contingencia se excita la oscilación del modo y a mayor flujo de potencia por el enlace mayor es la amplitud de la oscilación. Para ambos niveles de transferencia el amortiguamiento es positivo, indicando que el sistema es

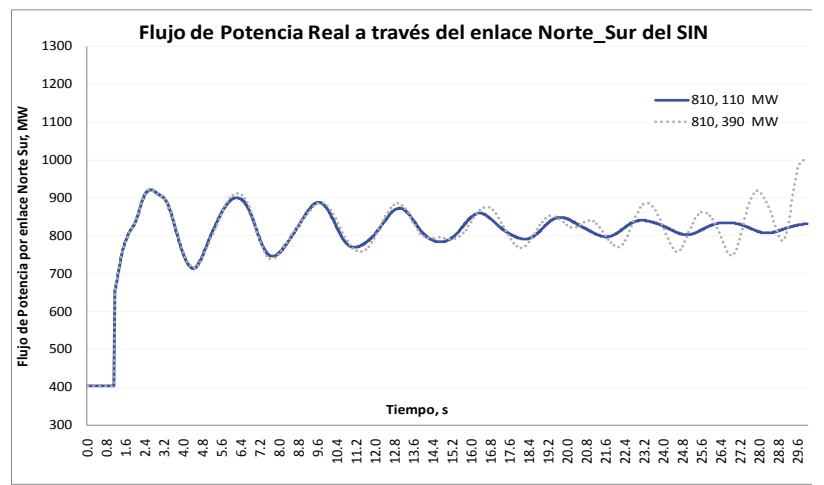

Figura 16. Comportamiento del modo de oscilación interárea Norte-Sur al incrementar el flujo por el enlace Peninsular estable. Estos resultados son congruentes con los obtenidos en los casos 1 y 2 .

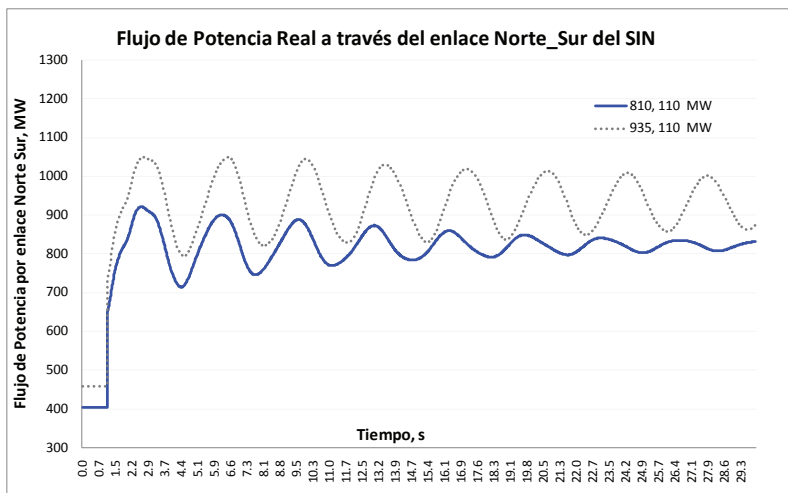

Figura 15. Comportamiento del modo de oscilación interárea Norte-Sur al incrementar el flujo por el enlace Norte-Sur

En la figura 16 se muestra el comportamiento dinámico del modo de oscilación interárea de $0.32 \mathrm{~Hz}$ ante el incremento del flujo de potencia de 110 a 390 MW a través del enlace Peninsular-Oriental. Se observa que ante la contingencia se excita la oscilación del modo. Para el nivel de flujo de potencia por el enlace PeninsularOriental de 110 MW el amortiguamiento es positivo. Sin embargo, cuando el flujo de potencia por el enlace Peninsular-Oriental de 390 MW el amortiguamiento es negativo indicando que el sistema es inestable. De estos resultados se puede deducir que los modos de oscilación del sistema eléctrico pueden tener cierto grado de interacción, lo que sugiere la realización de un análisis global en el que se consideren todas las dinámicas de la red eléctrica.

La figura 17 muestra el comportamiento dinámico del modo de oscilación interárea de $0.32 \mathrm{~Hz}$ ante el incremento del flujo de potencia de 810 a 935 MW a través del

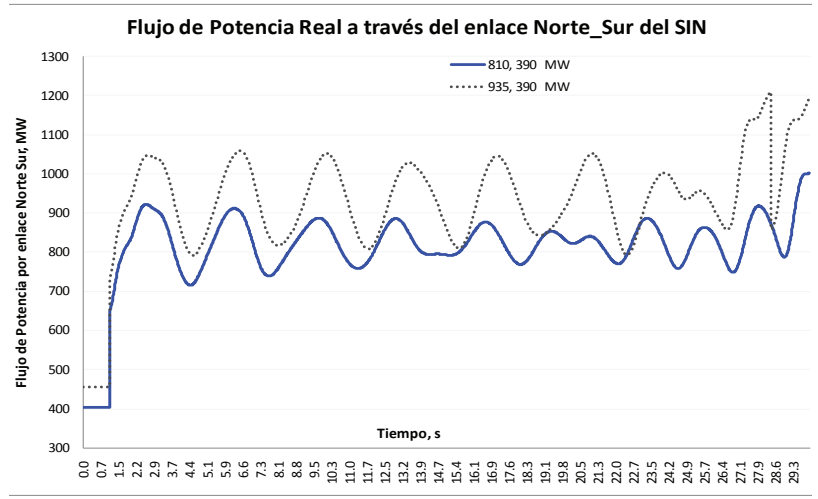

Figura 17. Comportamiento del modo de oscilación interárea Norte-Sur al incrementar el flujo por el enlace Norte-Sur y estresando flujo de potencia del enlace Peninsular 


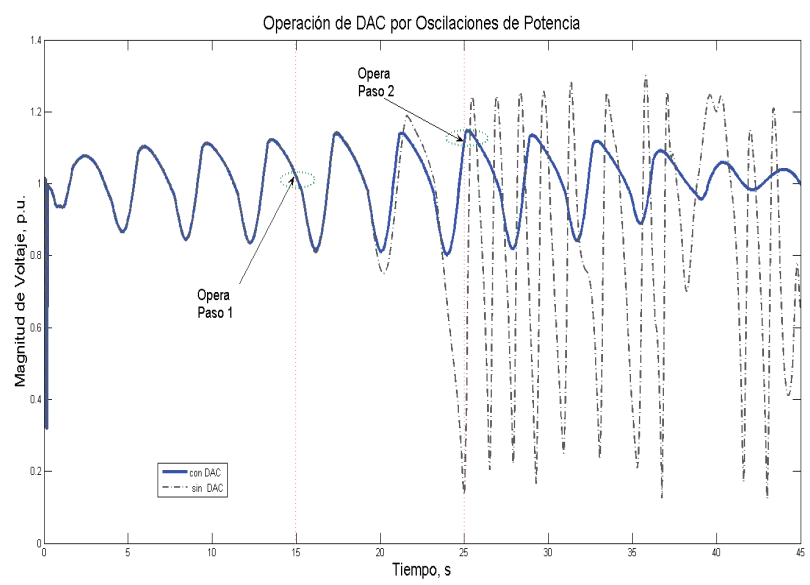

Figura 18. Operación de dos pasos del esquema de disparo automático de carga por oscilaciones de potencia

enlace Norte-Sur, pero a diferencia de la figura 16, se considera un mayor flujo por el enlace Peninsular-Oriental. Se observa que ante la contingencia se excita la oscilación del modo y a mayor flujo de potencia por el enlace mayor es la amplitud de la oscilación. Para ambos nive- les de transferencia el amortiguamiento es negativo indicando que el sistema es inestable. Estos resultados son congruentes con los obtenidos en los casos 3 y 4 , donde la inestabilidad está asociada al elevado nivel de transferencia por el enlace Peninsular-Oriental.

- Incremento de la seguridad operativa del sistema eléctrico de potencia. De los resultados anteriores se observa que hay una gran cantidad de incertidumbres que deben considerarse para mantener un comportamiento dinámico estable de las redes eléctricas. La determinación de límites de transferencia es una tarea compleja en la que entran en juego diversas políticas de operación (distintas para cada red eléctrica), los criterios de los diseñadores, la experiencia de operadores, entre otros. Lo anterior sugiere la necesidad de esquemas de protección automáticos como una acción de defensa contra posibles condiciones de operación inestables en los sistemas eléctricos de potencia.

Para el caso de oscilaciones interárea con amortiguamiento negativo se pueden utilizar esquemas de pro-

Tabla 2. Límite térmico, por cargabilidad y por oscilaciones interárea del enlace Norte-Sur

Tipo de Límite

Consideraciones y parámetros del enlace [ conductor ACSR Bluejay 1113 KCM ]
Límite por enlace con criterio n-1

(MW)
Térmico

Cargabilidad

Oscilaciones interárea

Estabilidad transitoria
Temperatura conductor: $75^{\circ} \mathrm{C}$

Temperatura ambiente: $45^{\circ} \mathrm{C}$ Velocidad del viento: $2 \mathrm{~m} / \mathrm{s}$ Ángulo del viento: $45^{\circ} \mathrm{C}$

Altitud: $350 \mathrm{msnm}$

Límite térmico del conductor: 1020 A, un conductor

2040 A, un circuito (dos conductores por fase)

$4080 \mathrm{~A}$, dos circuitos

Tensión de operación: 400 kV

Longitud del enlace : $275 \mathrm{~km}$ (Huinala-Champayan)

Dos conductores por fase

Voltaje plano (1 p.u.) en ambos extremos del enlace

$\mathrm{SIL}=522 \mathrm{MW}$

Diferencia del ángulo de voltaje entre extremos de enlace: $35^{\circ}$

Potencia reactiva absorbida, por la línea, de la red: 355 MVAR

Perdidas de Potencia: 43.8 MW, 294 MVAR.

No se considera compensación serie o paralelo en puntos intermedios del enlace.

Análisis lineal: valores propios, factores de participación, etcétera PSSs en todas las unidades de las plantas: CBD, SYU y MDP Indice de amortiguamiento: $\zeta>3 \%$

Análisis no-lineal: simulaciones en el tiempo, análisis de Prony
1414

$=\sqrt{3}\left(400 k V^{*} 2.04 k A\right)$
870

935

800 
tección automáticos (Castellanos et al., 2008) que realicen el disparo/desconexión de carga y/o generación ante oscilaciones con amortiguamiento negativo. La figura 18 muestra la aplicación de un esquema de corte de carga por oscilaciones de potencia. La contingencia crítica utilizada en el análisis es la salida de operación de un circuito del enlace Norte-Sur ante una falla trifásica en un extremo de la línea.

El esquema de corte de carga se activa al detectarse oscilaciones crecientes (negativas) en el punto de monitoreo del enlace asociado al modo de oscilación de 0.32 $\mathrm{Hz}$ operando dos pasos del esquema. En el primer paso, a los 15 segundos se desconectan 100 MW del nodo BUS A de carga. Como la oscilación continúa con amortiguamiento negativo se requiere la operación del segundo paso en el que, alrededor del segundo 25 se desconectan 85 MW de BUS B de carga. Posterior a la operación del segundo paso la oscilación presenta un amortiguamiento positivo, por lo que el esquema de control ya no dispara más carga.

Es importante destacar aquí que, en general, un corte de carga pequeño puede ser insuficiente para ayudar a mitigar las oscilaciones de potencia. Por otra parte, la desconexión de una gran cantidad de carga puede causar un serio e innecesario desbalance de potencia en el sistema. Por lo tanto, es importante seleccionar apropiadamente los montos y los sitios de carga a desconectar, así como la estrategia de control a utilizar, de tal manera que el esquema automático de corte de carga contribuya efectivamente al mejoramiento de la estabilidad del sistema de potencia.

\section{Límites de transferencia potencia del enlace Norte-Sur del Sistema Interconectado Mexicano}

En este inciso se muestra el límite térmico, el de cargabilidad y el obtenido ante oscilaciones interárea del en-

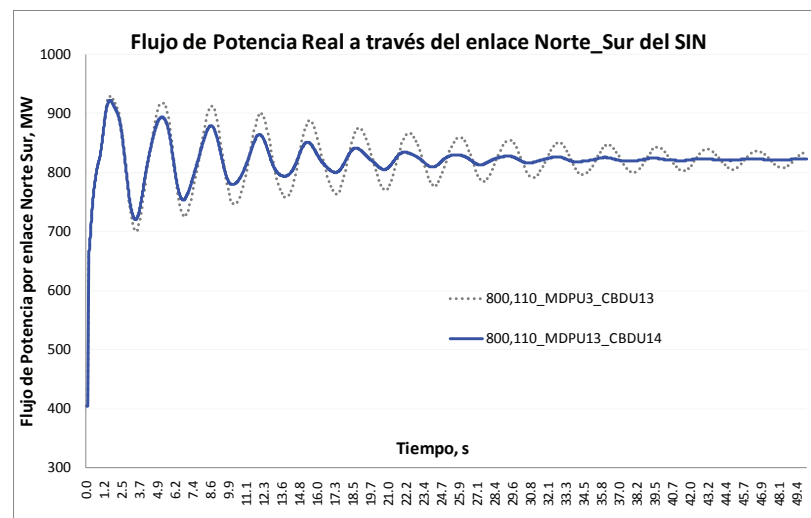

Figura 19. Comportamiento dinámico del flujo de potencia por el enlace Norte-Sur variando los PSS lace Huinala_Laja_Guemez_Champaya 400 kV, localizada en el enlace Norte-Sur del SIN. Las características de la línea de transmisión son las siguientes:

- Tensión de operación de 400 kV

- Conductor tipo ACSR, Bluejay, calibre 1113 KCM

- Dos circuitos, dos conductores por fase (cada circuito)

- Longitud del enlace: 275 k m (Huinala_Champayan)

En la tabla 2 se muestra el límite térmico por cargabilidad y ante oscilaciones interárea del enlace Norte-Sur indicando las consideraciones y parámetros utilizados en los cálculos. Para obtener los límites mostrados, en la tabla 2 , se considera el criterio n-1, es decir, el enlace opera con uno de sus dos circuitos o al estar operando con sus dos circuitos uno de ellos se desconecta.

Es importante indicar, que para la determinación del límite de transferencia por cargabilidad de la línea no se está considerando en el análisis compensación reactiva intermedia, la cual, puede extender este valor. Sin embargo, como el límite por oscilaciones interárea es muy cercano al de cargabilidad pudiera no ser de gran interés el incremento de este último.

Respecto al límite por oscilaciones interárea aplicando el análisis lineal, el valor de 935 MW se obtuvo considerando los PSS en las principales unidades con alta influencia en el comportamiento del modo de $0.32 \mathrm{~Hz}$ Norte-Sur, como se indica en el caso 8 de la tabla 1. En este caso, la relación de amortiguamiento es ligeramente superior a $11 \%$, manteniendo cierta reserva de amortiguamiento que puede ser de utilidad cuando alguno de los PSS de las plantas SYU, CBD y MDP esté fuera de operación y ante una contingencia múltiple.

El límite de transferencia de potencia obtenido mediante el análisis no-lineal de oscilaciones interárea, que se muestra en la tabla 2 , se obtuvo como se indica a continuación.

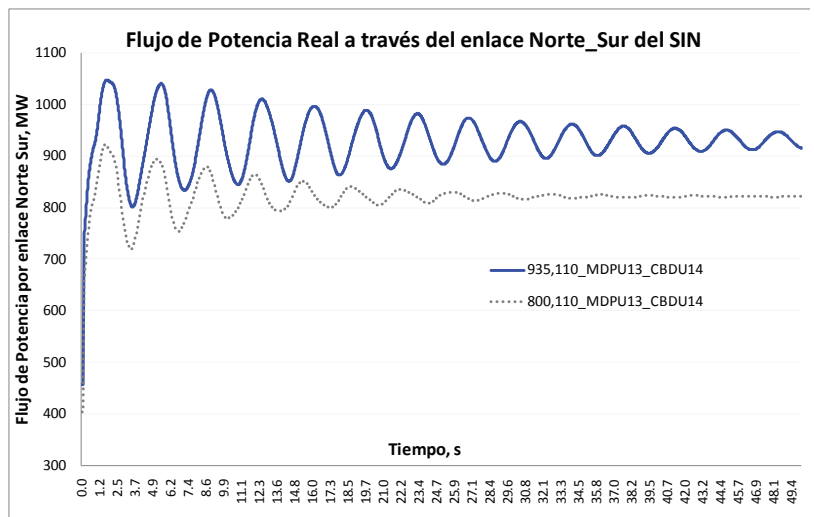

Figura 20. Comportamiento dinámico del flujo de potencia Norte-Sur variando el nivel de transferencia por el enlace 
a)

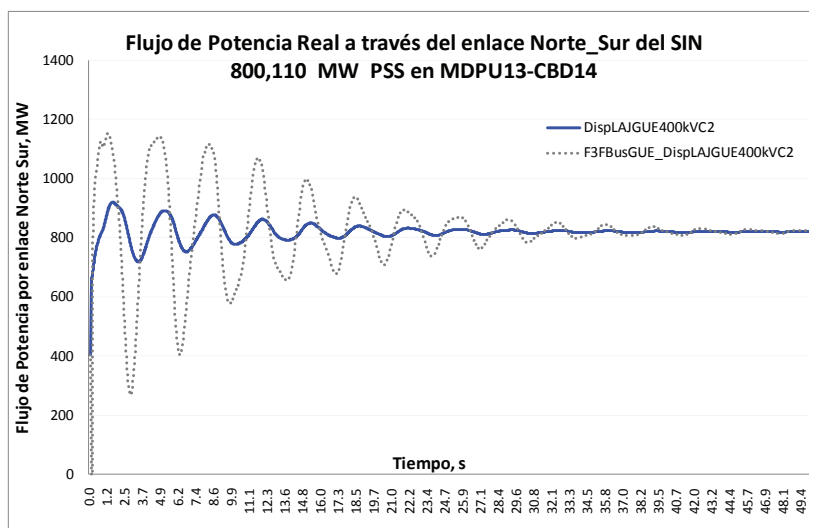

b)

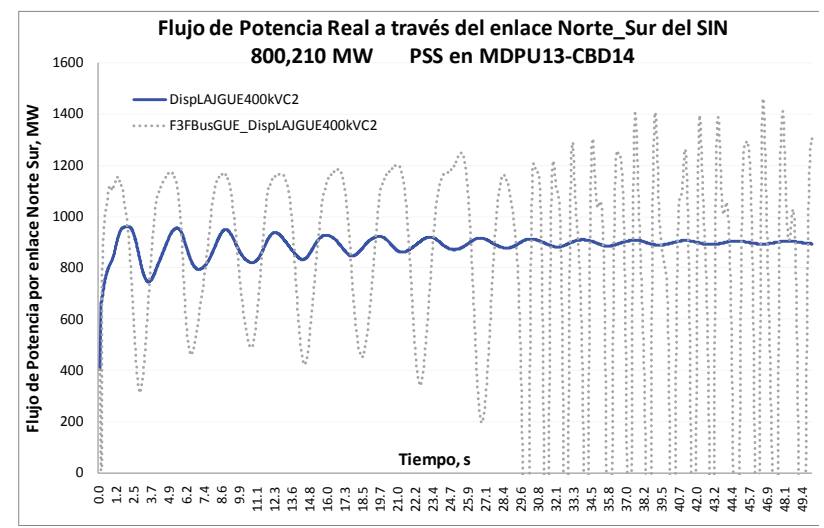

Figura 21. Comportamiento dinámico del flujo de potencia por el enlace Norte-Sur incrementando el flujo por el enlace peninsular para diversas contingencias, a) flujo de potencia medio por el enlace Peninsular-Oriental, b) flujo de potencia alto por el enlace Peninsular-Oriental

Tabla 3. Análisis de Prony de oscilaciones inter área del enlace Norte-Sur ante contingencias críticas con diversos PSS

\begin{tabular}{|c|c|c|c|c|c|}
\hline Flujo por enlace, MW & Ubicación de PSSs & Contingencia & $\begin{array}{l}\text { Estatus de } \\
\text { Seguridad }\end{array}$ & $\begin{array}{l}\text { Análisis de Prony } \\
\lambda * \\
(\zeta, f)^{* *}\end{array}$ & \\
\hline \multirow[t]{3}{*}{$\begin{array}{l}\text { Norte-Sur } 800 \\
\text { Peninsular } 110\end{array}$} & \multirow[t]{3}{*}{$\begin{array}{l}\text { CBD U1-U3 } \\
\text { MDP U3 }\end{array}$} & 1 & Seguro & $\begin{array}{l}-0.046 \pm \mathrm{j} 1.82 \\
(0.29,2.54)\end{array}$ & \\
\hline & & 2 & Inseguro & - & \\
\hline & & 3 & Inseguro & - & \\
\hline \multirow{3}{*}{$\begin{array}{l}\text { Norte-Sur } 800 \\
\text { Peninsular } 110\end{array}$} & \multirow{3}{*}{$\begin{array}{l}\text { CBD U1-U4 } \\
\text { MDP U1-U3 }\end{array}$} & 1 & Seguro & $\begin{array}{l}-0.104 \pm \mathrm{j} 1.83 \\
(0.29,5.64)\end{array}$ & \\
\hline & & 2 & Seguro & $\begin{array}{l}-0.104 \pm j 2.06 \\
(0.29,5.63)\end{array}$ & \\
\hline & & 3 & Seguro & $\begin{array}{l}-0.104 \pm \mathrm{j} 2.06 \\
(0.29,5.64)\end{array}$ & \\
\hline \multirow[t]{3}{*}{$\begin{array}{l}\text { Norte-Sur } \quad 800 \\
\text { Peninsular } 210\end{array}$} & \multirow[t]{3}{*}{$\begin{array}{l}\text { CBD U1-U4 } \\
\text { MDP U1-U3 }\end{array}$} & 1 & Seguro & $\begin{array}{l}-0.064 \pm \mathrm{j} 1.75 \\
(0.28,3.68)\end{array}$ & \\
\hline & & 2 & Inseguro & - & \\
\hline & & 3 & Inseguro & - & \\
\hline \multirow[t]{3}{*}{$\begin{array}{l}\text { Norte-Sur } \quad 800 \\
\text { Peninsular } 210\end{array}$} & \multirow{3}{*}{$\begin{array}{l}\text { CBD U1-U4 } \\
\text { SYU U1-U2 } \\
\text { MDP U1-U3 }\end{array}$} & 1 & Seguro & $\begin{array}{l}-0.111 \pm \mathrm{j} 1.75 \\
(0.28,6.3)\end{array}$ & \\
\hline & & 2 & Seguro & $\begin{array}{l}-0.111 \pm \mathrm{j} 1.75 \\
(0.28,6.8)\end{array}$ & \\
\hline & & 3 & Seguro & $\begin{array}{l}-0.122 \pm \mathrm{j} 1.74 \\
(0.28,6.9)\end{array}$ & \\
\hline \multirow[t]{3}{*}{$\begin{array}{lr}\text { Norte-Sur } & 935 \\
\text { Peninsular } & 110\end{array}$} & \multirow[t]{3}{*}{$\begin{array}{l}\text { CBD U1-U4 } \\
\text { MDP U1-U3 }\end{array}$} & 1 & Seguro & $\begin{array}{l}-0.044 \pm \mathrm{j} 1.75 \\
(0.28,2.50)\end{array}$ & \\
\hline & & 2 & Inseguro & - & * $\lambda$ es el valor \\
\hline & & 3 & Inseguro & - & $\begin{array}{l}\text { propio del modo de } \\
\text { oscilación, en } 1 / \mathrm{s} \text { y }\end{array}$ \\
\hline \multirow{3}{*}{$\begin{array}{l}\text { Norte-Sur } 935 \\
\text { Peninsular } 110\end{array}$} & \multirow{3}{*}{$\begin{array}{l}\text { CBD U1-U4 } \\
\text { SYU U1-U2 } \\
\text { MDP U1-U3 }\end{array}$} & 1 & Seguro & $\begin{array}{l}-0.099 \pm \mathrm{j} 1.74 \\
(0.28,5.71)\end{array}$ & $\begin{array}{l}\text { rad. } \\
* * \text { Relación de }\end{array}$ \\
\hline & & 2 & Inseguro & $\begin{array}{l}(0.28,5.71) \\
-\end{array}$ & amortiguamiento $(\zeta)$ \\
\hline & & 3 & Inseguro & - & (f) en $\mathrm{Hz}$ \\
\hline
\end{tabular}


La figura 19 muestra el comportamiento dinámico del flujo de potencia por el enlace Norte-Sur del SIN al variar el número los PSS conectados a máquinas con alta participación del modo de oscilación de $0.34 \mathrm{~Hz}$. Se observa que al aumentar el número de los PSS mejora el nivel de amortiguamiento de la oscilación de potencia.

La figura 20 muestra el comportamiento dinámico del flujo de potencia por el enlace Norte-Sur del SIN al incrementar el nivel de transferencia de potencia por el enlace indicando que entre mayor es el flujo de potencia menor es la relación de amortiguamiento del modo de oscilación de $0.34 \mathrm{~Hz}$.

La figura 21 muestra el comportamiento dinámico del flujo de potencia por el enlace Norte-Sur del SIN ante diversas contingencias para dos condiciones de flujo a través del enlace Peninsular-Oriental. Se observa que el tipo de contingencia tiene alta influencia en el comportamiento dinámico de la oscilación. Así, la figura $21 \mathrm{~b}$ indica que incrementando el flujo por el enlace Peninsular-Oriental el modo de oscilación de $0.34 \mathrm{~Hz}$ Norte-Sur la operación de la red eléctrica puede experimentar una condición de inestabilidad ante falla trifásica en el Bus Gue_400 kV con disparo de uno de los dos circuitos de la línea Laj_Gue_400 kV.

En la tabla 3 se muestran los resultados obtenidos al aplicar el análisis de Prony a las curvas que muestran el comportamiento dinámico de los flujos de potencia por los enlaces, con diversos PSS y ante varias contingencias, ver figuras 19-21. Las contingencias críticas consideradas en este análisis son:

1. Disparo sin falla de uno de los dos circuitos de la línea Laj_Gue_400 kV.

2. Falla trifásica en la línea Laj_Gue_400 kV con disparo de uno de los dos circuitos.

3. Falla trifásica en el Bus Gue_ $400 \mathrm{kV}$ con disparo de uno de los dos circuitos de la línea Laj_Gue_400 kV.

Los resultados de las simulaciones con el modelo no lineal del SIN indican 800 MW como límite máximo de transferencia por el enlace Norte-Sur, considerando la operación de por lo menos los PSS ubicados en las plantas de Carbón Dos (CBD) y Mérida Potencia Dos (MDP).

Los resultados mostrados en la tabla 2 indican que el límite térmico es de 2.7 veces el SIL de la línea eléctrica y está muy por arriba del resto, donde el de cargabilidad es 1.77, el de análisis lineal de oscilaciones inter área es 1.79 y el de análisis no-lineal de oscilaciones interárea es 1.5 veces el valor del SIL. Así, el análisis nolineal de oscilaciones interárea sugiere un flujo de potencia máximo de $800 \mathrm{MW}$ a través del enlace Norte-
Sur, para obtener un comportamiento dinámico adecuado de la red eléctrica.

\section{Conclusiones}

Las líneas de energía eléctrica de pequeña longitud podrían llegar a transmitir niveles de potencia cercanos a su límite térmico. Para el caso de líneas de mayor longitud existen restricciones como son cargabilidad y estabilidad que limitan la transferencia de potencia a valores menores a su límite térmico. Cuando el límite de transferencia de un enlace es térmico, se puede incrementar la capacidad de transferencia de estos utilizando conductores de alta temperatura.

El límite por cargabilidad de una línea eléctrica depende de factores como: la tensión de operación, longitud de la línea y número de conductores por fase, fuentes de compensación de potencia reactiva que incrementan la capacidad de transferencia y/o proporcionan un mayor soporte de voltaje.

El límite de estabilidad debido a oscilaciones inter área de un enlace de transmisión de energía eléctrica está en función de una gran cantidad de variables como son: el nivel de flujo de potencia por enlaces asociados a los modos de oscilación, la impedancia equivalente de estos enlaces, el número de máquinas síncronas con PSS, otras fuentes de amortiguamiento proveniente del control suplementario de los compensadores estáticos de vars. Para tener una operación segura de la red eléctrica ante oscilaciones interárea con amortiguamiento negativo se pueden utilizar esquemas de protección automáticos que realicen el disparo/desconexión de carga y/o generación ante oscilaciones con amortiguamiento negativo.

\section{Referencias}

Castellanos R.B., Messina A.R., Sarmiento H., Pampin G. Assessment of Remedial Control Schemes for Damping Transient Oscillations in the Mexican System, en: Cigre Canada, Conference on Power Systems, octubre 2008, Winnipeg, Can.

Castellanos R.B., Messina A.R., Calderon J.GG., Sarmiento H.U. Large-Scale Use of FACTS Technology for Damping InterArea Oscillations in the Mexican System, en: IEEE PES General Meeting (2007, Tampa, Florida, USA.

Castellanos R.B., Calderon J.G.G., Olguin D.S., Sarmiento H.U., Messina A.R. Use of Power System Stabilizers for Damping Inter-Area Oscillations in the South Systems of the Mexican Electrical Grid, en: Electric Power Systems Research 76(2006), enero de 2006, pp. 169-179.

CFE E0000-12. Cables de aluminio con cableado concentrico y alma de acero (ACSR), México, CFE, mayo 1996. 
Castellanos R.B., Messina A.R., Calderon J.GG., Sarmiento H.U. Large-Scale Use of FACTS Technology for Damping InterArea Oscillations in the Mexican System, en: IEEE PES General Meeting (2007, Tampa, Florida, USA.

Castellanos R.B., Calderon J.G.G., Olguin D.S., Sarmiento H.U., Messina A.R. Use of Power System Stabilizers for Damping Inter-Area Oscillations in the South Systems of the Mexican Electrical Grid, en: Electric Power Systems Research 76(2006), enero de 2006, pp. 169-179.

CIGRE, Analysis and Control of Power System Oscillations, CIGRE, Task Force 07 of Advisor Group 01 of Study Committee 38, Paris, diciembre de 1996.

CIGRE. Considerations Relating to the Use of High Temperature Conductors, B2 WG03, B2 WG12, B2 WG11. Paris: 331, octubre 2007.

Duncan-Glover J., Sarma M. Power System Analysis and Design, second edition, PWS Publishers, 1990.

IEEE Std 738-2002. IEEE Standard for Calculating the Current Temperature Relationship of Bare Overhead Conductors, New York, IEEE, 2002.

Kundur P. Power System Stability and Control, first edition, McGraw-Hill, Inc, 1994.

Kyeon H., Boddeti M., Sarma N.D.R., Dumas J., Adams J., SoonKin C. High-Wire Act. IEEE Power \& Energy Magazine for Elec- tric Power Professionals, volumen 8 (número 1), enero-febrero de 2010.

Powertech Labs Inc. Small Siganl Stability Assessment Tool, SSAT, Version 7.0, mayo 2007.

Powertech Labs Inc. Transient Security Assessment Tool, TSAT, Version 7.0, mayo 2007.

System Oscillation Working Group. Inter-Area Oscillations in Power Systems, en: IEEE 95 TP 101, Piscataway, NJ, USA, 1995.

Westinghouse Electric Corporation. Electrical Transmission and Distribution Reference Book, Characteristics of Aerial Lines, fourth edition, East Pittsburgh, PA, septiembre 1950, p. 49.

\section{Este artículo se cita:}

\section{Citación estilo Chicago}

Castellanos-Bustamante, Rafael. Determinación de límites de transmisión en sistemas eléctricos de potencia. Ingeniería Investigación y Tecnología, XV, 02 (2014): 271-286.

\section{Citación estilo ISO 690}

Castellanos-Bustamante R. Determinación de límites de transmisión en sistemas eléctricos de potencia. Ingeniería Investigación y Tecnología, volumen XV (número 2), abril-junio 2014: 271-286.

\section{Semblanza del autor}

Rafael Castellanos-Bustamante. Ingeniero eléctrico por la Universidad Autónoma del Estado de Morelos. Recibió el grado de maestro en ciencias en ingeniería eléctrica por el Instituto Politécnico Nacional en 1997 y el grado de doctor en ciencias por el Centro de Investigación y Estudios Avanzados del IPN en 2006. A partir de 1994, labora para la gerencia de transmisión y distribución del Instituto de Investigaciones Eléctricas de México. Sus principales áreas de interés son el diseño y análisis dinámico de sistemas eléctricos de potencia. 